\title{
Time-Accurate Navier-Stokes Calculations with Approximately Factored Implicit Schemes
}

\author{
Richard P. Dwight \\ German Aerospace Center (DLR), Braunschweig, Germany. \\ richard.dwight@dlr.de
}

Summary. The performance of the dual time method is improved by means of an inner iteration based on an LU-SGS driven FAS multigrid method, and by using the same LU-SGS method in a time-accurate mode to provide a starting solution for each dual time step. This hybrid scheme is applied to an aerofoil undergoing dynamic stall, whereby considerable performance improvements are demonstrated over the original dual time method.

\section{Introduction}

One of the most computationally demanding tasks in modern CFD is the simulation of unsteady flows. The lack of efficient algorithms for non-stationary problems either limits the accuracy to which cases may be solved, or creates the demand for more computing resources than the engineer is able to provide.

Typically modern flow solvers tackle unsteady problems by employing a fully implicit time discretization, in which the non-linear system is solved by an inner iteration, the dual time method [4]. The inner iteration strongly resembles the iteration for convergence to steady state, so that all techniques developed for efficient steady state convergence (most of which sacrifice time accuracy) may be applied. However even with the application of local time stepping, FAS multigrid, residual smoothing and an explicit Runge-Kutta scheme, typically 50-100 inner iterations are necessary for convergence. In the case that particularly sensitive integrated quantities are of interest, for example the rolling moment of a delta wing, even more iterations are required.

The alternative approach, of linearizing the implicit time discretization and solving the resulting linear system at each time step, has the serious disadvantage that the linear system must be constructed and solved exactly if time accuracy is to be preserved. Most practical implicit solvers on the other hand make use of an approximate system matrix and an inexact linear solver to reduce memory requirements and remain competitive with the efficiency of explicit solvers [1]. Even if everything is formulated exactly, the linearization limits the time accuracy to at most second order.

In this paper a combination of a dual-time method with a first order time accurate LU-SGS method [7] is presented. The hybrid scheme resembles the proposed scheme of Hsu et al. [3] in that an initial approximate implicit step provides a starting solution for a dual time inner iteration. The convergence behavior of the inner iteration is improved by applying LU-SGS as a multigrid smoother. The use 
of LU-SGS ensures that the memory requirements of the implicit solver remain low. A performance improvement over the dual time scheme when used in isolation is demonstrated for a two-dimensional dynamic stall test case, for an implementation in the DLR unstructured solver TAU [2].

\section{Theory}

The governing equations are the Reynolds Averaged Navier-Stokes (RANS) equations closed by a one- or two-equation turbulence model. The computational domain is discretized by an unstructured grid with a cell-vertex metric, and the finite volume method is applied. It is assumed for simplicity that the grid undergoes only rigid body motion.

\subsection{Temporal Discretization}

Consider the semi-discretization of the governing equations

$$
\frac{\mathrm{d} W_{i}}{\mathrm{~d} t}+R_{i}(W)=0
$$

where the subscript $i$ indicates the index of a point in the grid, and the residual $R$ contains contributions from both fluid fluxes and whirl fluxes due to the motion of the grid. Discretize (1) in time by means of the generalized trapezoidal scheme

$$
\Omega_{i} \frac{W_{i}^{(n+1)}-W_{i}^{(n)}}{\Delta t_{i}}=-\beta R_{i}\left(W^{(n+1)}\right)-(1-\beta) R_{i}\left(W^{(n)}\right) .
$$

which is first order accurate in time for $\beta=1$ and second order accurate for $\beta=\frac{1}{2}$. For both values of $\beta$ the scheme is A-stable. It has the advantage over backward difference formulae (BDF) that the solution at time levels $n-1$ and lower are not needed.

\subsection{Linearization of the Implicit Scheme}

To reduce the non-linear algebraic system of (2) to a linear system it is necessary to linearize $R_{i}\left(W^{(n+1)}\right)$ for each grid point about the known solution $W^{(n)}$. This follows as

$$
\begin{aligned}
R_{i}\left(W^{(n+1)}\right) & =R_{i}\left(W^{(n)}\right)+\frac{\partial R_{i}\left(W^{(n)}\right)}{\partial t} \Delta t+\mathcal{O}\left(\Delta t^{2}\right), \\
& =R_{i}\left(W^{(n)}\right)+\sum_{j \in \mathcal{N}(i)} \frac{\partial R_{i}\left(W^{(n)}\right)}{\partial W_{j}} \frac{\partial W_{j}}{\partial t} \Delta t+\mathcal{O}\left(\Delta t^{2}\right),
\end{aligned}
$$

where $\mathcal{N}(i)$ is the set of grid points in the stencil of $R_{i}$. Substituting (4) into (2), and applying

$$
\frac{\partial W_{j}}{\partial t} \Delta t=\Delta W_{j}+\mathcal{O}\left(\Delta t^{2}\right)
$$

results in the linear algebraic system 


$$
\left\{\frac{\Omega_{i}}{\Delta t_{i}} \delta_{i j}+\beta \frac{\partial R_{i}\left(W^{(n)}\right)}{\partial W_{j}}\right\} \cdot \Delta W_{j}^{(n)}=-R_{i}\left(W^{(n)}\right),
$$

where the summation convention is used on $j$. Note that the linear system takes the same form for first and second order time accuracy, allowing a general implementation of linear solver and left-hand side evaluation. This expression retains the second order time accuracy of (2) for $\beta=\frac{1}{2}$ provided that the flux Jacobian of the discrete equations $\partial R / \partial W$ is evaluated exactly. Otherwise the method is first order accurate, with leading order error controlled by the approximation of the flux Jacobian.

\subsection{Solution of the Linear System by LU-SGS}

The LU-SGS method is applied to equation (6) to obtain an approximation for $\Delta W$. Rather than solving the original system, which may be written $A \cdot x=b$, an approximately factorized system, in which the system matrix is replaced by

$$
A^{\prime}=(L+D) D^{-1}(U+D) \approx(L+D) D^{-1}(U+D)-L D^{-1} U=A
$$

where $L, D$ and $U$ are the block lower triangular, diagonal and upper triangular parts of $A$, is solved. The solution operation may be performed using two GaussSeidel sweeps, equivalent to two triangular system solves, one lower and one upper. The error introduced due to the approximate factorization is $L D^{-1} U \Delta W \approx$ $\mathcal{O}\left(\Delta t^{2}\right)$.

A second approximation is to use a flux Jacobian based on first order fluxes in (6). These two approximations in combination have the important consequence of allowing the Gauss-Seidel sweeps to be performed without explicit storage of the system matrix [6], reducing the memory requirements to that of an explicit scheme. Furthermore a complete step may be performed in a time equivalent to that of a 3-stage Runge-Kutta step.

At this point there are four sources of error in the time discretization:

- the discretization error of the trapezoidal scheme,

- the truncation error of the linearization of $R\left(W^{(n+1)}\right)$,

- the factorization error of the LU-SGS scheme $L D^{-1} U \Delta W$,

- the error due to the first order approximation of the flux Jacobian.

All these errors, with the exception of the last, are nominally of size $\mathcal{O}\left(\Delta t^{2}\right)$, but the cost of increasing the accuracy of the last to second order is high in terms of memory and storage on unstructured grids. This approach leads to the high-power implicit methods discussed in Sect. 1.

\subsection{Dual Time Stepping}

Dual time is an alternative means of time integration. The time derivative of (1) is discretized with a BDF for example, and the resulting algebraic system is solved by iterating the equation

$$
\frac{\partial \bar{W}}{\partial \tau}=-\left\{\frac{\Omega\left(3 \bar{W}-4 W^{(n)}+W^{(n-1)}\right)}{2 \Delta t}+R(\bar{W})\right\}=-\bar{R}(\bar{W})
$$


to a steady state in $\tau$. Here $\tau$ is an artificially introduced pseudo time, $\bar{W}$ is the iterate, and the iterations are henceforth known as inner iterations. At a steady state $\partial \bar{W} / \partial \tau=0$ and hence $\bar{W}=W^{(n+1)}$. This system strongly resembles the original problem (1) for the steady state case, and so methods developed for the solution of that problem may be applied here also. Implicit methods are particularly suited to inner iterations as the extra term in the modified residual $\bar{R}$ increases the diagonal dominance of the flux Jacobian, improving the stability of e.g. LU-SGS.

\subsection{Hybrid Time Stepping}

At each step, dual time requires an initial estimate of the solution for that step, $\bar{W}^{0}$. This is typically provided by polynomial extrapolation of the solution at previous time levels to the new time level, $\bar{W}^{0}=W^{(n)}+\left(W^{(n)}-W^{(n-1)}\right)$, for linear extrapolation.

An alternative means of start-up might be to apply the LU-SGS method of Sect. 2.3 once in real time. This method has been demonstrated to be nominally first order in time, which is typically insufficient for direct use, but which may lead to a more accurate $\bar{W}^{0}$. LU-SGS has the ability to perform large steps stably, so the time step size will not be limited by the start-up step, and as seen in Sect. 2.2, the algorithm can be run in time accurate mode without modification, allowing very straightforward implementation. The nature of dual time means that the hybrid scheme will have the higher order time properties of the original BDF, provided inner iterations converge.

In what follows, dual time refers to (8) with linear extrapolation start-up, solved by explicit Runge-Kutta smoothed FAS multigrid iterations. Hybrid time refers to (8) with start-up by one or more iterations of LU-SGS with $\beta=\frac{1}{2}$, solved by LU-SGS smoothed multigrid with $\beta=1$.

\section{Results}

The algorithms of the preceeding sections have been implemented in the unstructured grid, compressible RANS solver TAU [2], which is developed at the German Aerospace Center (DLR). A dual time algorithm and general rigid body motion were already available in this code, and these enabled the rapid implementation and testing of the hybrid method.

The first test case is designed to determine the time accuracy of the LU-SGS scheme alone. This will help decide at what range of $\Delta t$ the hybrid method is likely to be effective. It is essential that this test be viscous, as the approximation of the viscous terms on the LHS of (6) will affect the time accuracy, as will the stretched boundary-layer cells of a viscous grid which will give locally very small $\Delta x$.

A harmonically oscillating RAE2822 aerofoil is chosen, with pitch angle $\alpha=$ $3^{\circ} \pm 2.51^{\circ}$, at a reduced frequency of $k=\omega l_{k} / V_{\infty}=0.163$. Under these conditions the flow remains fully attached at all stages of the motion. A third order accurate dual time method, with 200 inner iterations per time step and 1024 time steps per period (TSPP), was used to obtain a reference solution. Unsteady solutions obtained with LU-SGS alone, and second order accurate dual time alone, for varying 


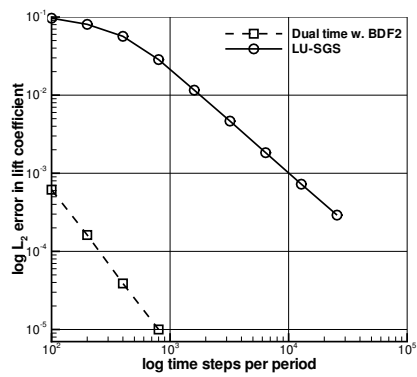

Fig. 1. Comparison of the time accuracy of second order dual time with LU-SGS.

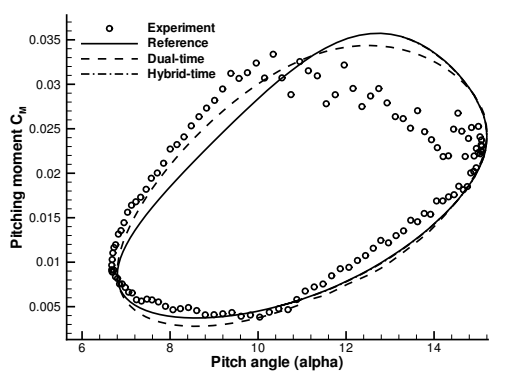

Fig. 2. Pitching moment of the NACA0015 test case. The test schemes use 30 inner iterations.

$\Delta t$ were compared with the reference solution using the $L_{2}$ norm of the difference in calculated lift coefficients over one period. The results are plotted in Fig. 1.

Both the second order behavior of dual time and the first order behavior of LUSGS are readily apparent, confirming the theory of Sec. 2.3. However the breakdown of the time accurate behavior of LU-SGS occurs at relatively large values of TSPP (about 300), which is a result of the $\Delta x$ dependence of the factorization error in very thin viscous wall cells. Further note that, in terms of absolute error, LU-SGS reaches the accuracy of 100 TSPP dual time with 10,000 TSPP. Since one dual time inner iteration costs as much as one LU-SGS step, the two methods have roughly equal efficiency (assuming 100 dual time inner iterations per step) for this level of accuracy.

The second test case was chosen to be a demanding problem of practical interest where polynomial extrapolation performs poorly. Dynamic stall refers to unsteady flow separation occurring on aerofoils executing unsteady motion, and is of great importance in rotorcraft aerodynamics where it can have a significant effect on the flight envelope of helicopters. The case considered here is that of a 2d NACA0015 aerofoil pitching harmonically with an angle of $\alpha=11^{\circ} \pm 4.2^{\circ}$ and a reduced frequency of $k=0.2$, being one of the cases investigated experimentally by Piziali [5].

Calculations were performed with the dual- and hybrid-time schemes with only 30 inner iterations, the results can be seen in Fig. 2, compared with a reference calculation using 200 inner iteration dual time, and experimental results. While the dual time scheme deviates from the reference result significantly, the hybrid scheme shows no such difference. The cause can be seen in the convergence of the inner iterations for the two schemes, shown in Fig. 3. Integrated values for dual time do not converge within the 30 iteration limit. Corresponding results have been obtained in three dimensions.

One question raised by hybrid time is, how many start-up LU-SGS steps should be taken for optimal performance? Shown in Fig. 4 is a hybrid scheme with 10 startup steps each taking one-tenth the time step of the outer scheme, followed by 20 continuation steps. Measurements of the time accuracy of the hybrid scheme for varying numbers of start-up steps produce the rule of thumb that about $10 \%$ of the total steps should be start-up steps. 


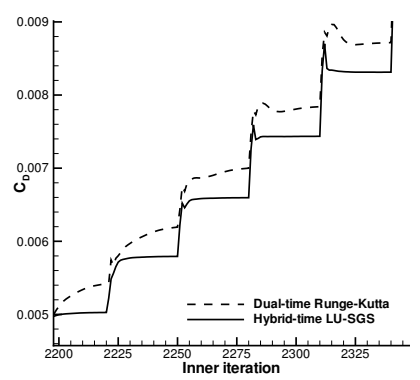

Fig. 3. Convergence for dual- and hybrid-time for 30 inner iterations.

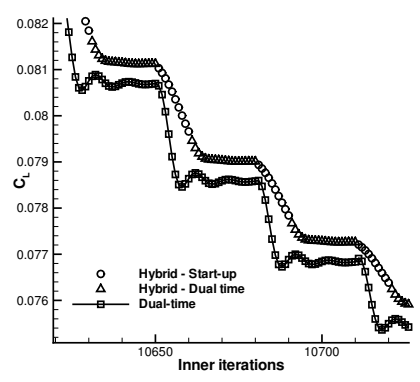

Fig. 4. Demonstration of the combination of LU-SGS with dual-time.

\section{Conclusion}

An original time stepping method has been introduced based on a combination of a nominally first order time accurate formulation of the LU-SGS implicit scheme, and a dual time method driven by LU-SGS inner iterations. This hybrid scheme has been implemented in an unstructured grid RANS code and applied to dynamic stall on a two-dimensional aerofoil. It has been found to offer moderate efficiency improvements over the dual time scheme used in isolation.

To improve the efficiency of the hybrid scheme further it will be necessary to improve the time accuracy of the LU-SGS start-up step. This may be accomplished with a better flux Jacobian approximation.

\section{References}

1. T. Chisholm and D.W. Zingg. A fully coupled Newton-Krylov solver for turbulent aerodynamic flows. ICAS 2002 Conference, Toroto, (Paper 333), 2002.

2. T. Gerhold, M. Galle, O. Friedrich, and J. Evans. Calculation of complex 3D configurations employing the DLR TAU-Code. AIAA Paper, (AIAA-97-0167), 1997.

3. J.M. Hsu and A. Jameson. An implicit-explicit hybrid scheme for calculating complex unsteady flows. Proceedings of 40th AIAA CFD Conference, Reno, (AIAA-2002-0714), 2002.

4. A. Jameson. Time dependant calculations using multigrid with applications to unsteady flows past airfoils and wings. AIAA Paper, (AIAA-91-1596), 1991.

5. R.A. Pizialli. An experimental investigation of $2 \mathrm{D}$ and $3 \mathrm{D}$ oscillating wing aerodynamics for a range of angle of attack including stall. NASA Technical Memorandum, (4632), 1993.

6. D. Sharov and K. Nakahashi. Reordering of hybrid unstructured grids for LowerUpper Symmetric Gauss-Seidel computations. AIAA Journal, 36(3):484-486, 1997.

7. S. Yoon and A. Jameson. An LU-SSOR scheme for the Euler and Navier-Stokes equations. AIAA Journal, 26:1025-1026, 1988. 